\title{
Los padres como promotores del desarrollo de lenguaje de bebés prematuros: propuesta de intervención temprana
}

\author{
Parents as Promoters of Language Development in \\ Premature Babies: a Proposal for Early intervention
}

\author{
Melissa Calderón-Carrillo ${ }^{1}$ \\ Josefina Ricardo-Garcell ${ }^{2}$ \\ Lauren M. Cycyk ${ }^{3}$ \\ Donna Jackson-Maldonado ${ }^{4}$ \\ Gloria Avecilla-Ramírez ${ }^{5}$ \\ Thalía Harmony ${ }^{6}$ \\ 1,2,6 Universidad Nacional Autónoma de México, México \\ ${ }^{3}$ University of Oregon, United States \\ ${ }^{4,5}$ Universidad Autónoma de Querétaro, México
}

\begin{abstract}
Resumen. Cualquier programa de intervención puede beneficiarse de la participación de los padres, pero es necesario darles información de cómo pueden estimular el lenguaje de sus hijos. El objetivo fue entrenar a los padres acerca de lo que debían esperar del desarrollo del lenguaje de sus hijos y brindarles técnicas de estimulación de este para evitar secuelas. Se estudiaron 21 bebés prematuros mexicanos y se comprobó que, después del entrenamiento, los padres se sentían más capaces de estimular a sus hijos. A los bebés se les aplicó una prueba de lenguaje al año y a los tres años de edad; en ambas obtuvieron puntajes normales para su edad. Se concluye que los padres son buenos promotores del lenguaje en bebés prematuros.
\end{abstract}

Palabras clave. Intervención temprana, lenguaje, prematurez, desarrollo del lenguaje, educación de padres.

Abstract. Any program of intervention can benefit from the participation of parents. It is necessary to give parents information about what they can do to stimulate the language of their children. This paper aimed to train parents on what to expect in the language development of children, and to teach them techniques of stimulation with the purpose of preventing negative consequences in language development. The sample consisted of 21 premature babies. It was found that, after the training, the parents felt more confident in facilitating the language of their children. The children's language abilities at 12 months and 36 months were tested. The children obtained typical scores. It was concluded that parents are good promoters of language development in premature babies.

Keywords. Early intervention, language, prematurity, language development, parent education.

\footnotetext{
${ }^{1}$ Melissa Calderón-Carrillo. Universidad Nacional Autónoma de México, México. E-mail: mels44@gmail.com ${ }^{2} J o s e f i n a$ Ricardo-Garcell. Universidad Nacional Autónoma de México, México. Dirección postal: Campus Juriquilla, Querétaro, Boulevard Juriquilla, Querétaro, México. C.P. 76230. E-mail: oojrg@yahoo.com ${ }^{3}$ Lauren M. Cycyk. University of Oregon, United States. E-mail: lcycyk@uoregon.edu ${ }^{4}$ Donna Jackson-Maldonado. Universidad Autónoma de Querétaro, México. E-mail: djackson@uaq.mx ${ }^{5}$ Gloria Avecilla-Ramírez. Universidad Autónoma de Querétaro, México. E-mail: gloria.avecilla@uaq.mx ${ }^{6}$ Thalía Harmony. Universidad Nacional Autónoma de México, México. E-mail: thaliah@unam.mx
}

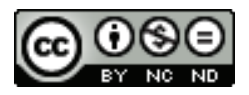

Esta obra está bajo una licencia de Creative Commons Reconocimiento-NoComercial-SinObraDerivada 4.0 Internacional. 


\section{Introducción}

En los últimos años ha aumentado el número de recién nacidos prematuros que sobreviven gracias a los avances en obstetricia y neonatología (Jiménez, Servera, Roca, Frontera, \& Pérez, 2008). Esta mayor incidencia de neonatos prematuros preocupa tanto al personal médico como a los familiares de los niños por las posibles secuelas en su desarrollo evolutivo. Los recién nacidos prematuros presentan, con frecuencia, bajo peso, un menor perímetro cefálico y mayores deficiencias motoras, cognitivas y lingüísticas que los bebés de término (Doyle, 2001).

Se ha observado que los bebés nacidos con bajo peso al nacer tienen mayores posibilidades de tener dificultades en el desarrollo de la percepción visual y visomotora, retraso en algunas funciones del lenguaje, deficiencias en la memoria de trabajo y, en la edad escolar, pueden presentar problemas de aprendizaje y déficit de atención (Horwood, Mogridge, \& Darlow, 1998). Sin embargo, la evidencia no es concluyente en cuanto al desarrollo del lenguaje, ya que los estudios comparativos acerca de la adquisición temprana del lenguaje en infantes prematuros y nacidos a término han reportado resultados controversiales.

Mientras algunos estudios han encontrado que los bebés prematuros tienen un retraso en sus habilidades de lenguaje comparados con bebés nacidos a término (Magill-Evans \& Harrison, 1999; 2001; Sansavini, Guarini, Alessandroni, Faldella, Giovanelli, \& Salvioli, 2006; Foster-Cohen, Edgin, Champion, \& Woodward, 2007; Kern \& Gayrau, 2007; OrtizMantilla, Choudhury, Leevers, \& Benasich, 2008; Wolke, Samara, Bracewell, \& Marlow, 2008; Guarini et al., 2009; Stolt, Haataja, Lapinleimu, \& Lehtonen, 2009; Stolt, Lehtonen, Haataja, \& Lapenleimu, 2011), en otros no se han encontrado diferencias significativas al comparar ambos grupos (Menyuk et al., 1991; Cattani et al., 2010; Pérez-Pereira \& Resches, 2011) cuando se hace una corrección de la edad y se iguala el nivel cognoscitivo.

Por otra parte, desde hace varias décadas, distintos autores han descrito que la intervención temprana en el área de la audición y del lenguaje, en niños con riesgo de manifestar dificultades en su desarrollo comunicativo, mejora una amplia gama de habilidades comunicativas y cognoscitivas (Barrera, Rosenbaum, \& Cunningham, 1986; Resnick, Eyler, Nelson Eitzman, \& Bucciarelli, 1987). Se ha demostrado que cuando se lleva a cabo una intervención temprana, los bebés prematuros se benefician positivamente; es decir, las brechas en el desarrollo se reducen considerablemente (Guralnick, 2008; 2012). Los programas de intervención temprana que enfocan sus esfuerzos tanto en los niños como en los padres son más efectivos que los que se apoyan solamente en el niño (Barrera, et al., 1986; Shonkoff \& Hauser-Cram, 1987).

¿Por qué los padres son una fuente de estimulación ideal para el desarrollo del lenguaje de sus hijos? Porque se sabe que la interacción social y las propiedades del habla adultoniño son de enorme influencia en la adquisición del lenguaje en los niños (Kuhl, 2014).

Se conoce que el cerebro del niño tiene una predisposición natural para la adquisición del lenguaje pero, recientemente, se ha llamado la atención hacia la posibilidad de que la interacción social promueva de manera poderosa el aprendizaje de la lengua en el niño (Kuhl, 2007). Por ejemplo, se ha encontrado que las características del habla adultoniño, también conocida como materlalia (frases cortas y sencillas, entonación exagerada, 
repetición y velocidad lenta) están asociadas con el aumento de la atención, la percepción, la comprensión y la conducta del niño (Snow, 1977; Fernald, 1993; Tsao, Liu, \& Kuhl, 2004; Liu, Tsao, \& Kuhl, 2009).

Pero no se trata solo de exponer al niño al habla de los padres, se requiere también de la interacción social. Se ha descrito que los niños pequeños producen más vocalizaciones cuando sus madres retroalimentan sus balbuceos con atenciones y sonrisas (Goldstein, King, \& West, 2003) e, incluso, son capaces de aprender mejor las categorías fonéticas de una lengua en presencia de interacciones sociales (Kuhl, 2007; Conboy, Brooks, Meltzoff, \& Kuhl, 2015). Por tanto, puede considerarse que el lenguaje utilizado en el contexto de las interacciones niño-adulto y que se caracteriza por la capacidad de respuesta y la retroalimentación positiva, sirven de andamiaje para el aprendizaje del niño y estimula sus conductas verbales (Zauche, Thul, Darcy, \& Staple-Wax, 2016).

El entrenamiento específico con los padres puede mejorar su sensibilidad y habilidad para estimular efectivamente a sus hijos en cuanto al desarrollo de sus procesos cognitivos (Barrera, et al., 1986). La intervención temprana basada en la exposición repetida a una estimulación sensorial coherente mejorará la posibilidad de generalizar la información y cambiar el comportamiento (Tallal \& Gabb, 2006).

A su vez, existe la posibilidad de que los niños prematuros presenten dificultades para mantener la atención a un estímulo auditivo específico, especialmente si existe un ruido de fondo. Ward (1999) comprobó que niños con retraso en el desarrollo del lenguaje podían ser equiparados al nivel comunicativo de sus pares de edad cronológica después de animar a sus padres a destinarles un tiempo, en un lugar tranquilo sin ruido y enseñarles como dirigir la atención a un sonido específico en el medio ambiente. Aunado a esto, también es probable que los padres estimulen a sus bebés prematuros no solamente en la atención auditiva sino también en otras áreas que son importantes para un desarrollo adecuado del lenguaje.

Dado que el lenguaje es inicialmente aprendido a través del modelo e interacción, los padres de los bebés prematuros son precisamente los más indicados para brindar una estimulación constante a sus bebés, esto porque los niños aprenden al ver a aquellos que están a su alrededor, imitándolos y después practicando con ellos. Por tanto, la familia de un niño es el mejor recurso para el contacto humano y la observación; el cómo interactúa la familia con el niño y cómo está estructurada, juegan un rol importantísimo en el desarrollo del lenguaje en un niño (Roberts \& Kaiser, 2011). En este sentido, los padres deben disponer de información adecuada, desde el punto de vista técnico, que les permita contar con las mejores herramientas para potenciar el desarrollo de sus hijos y, en particular, su lenguaje.

El presente trabajo tiene como objetivo mostrar los beneficios de una propuesta de intervención temprana, basada en la educación de los padres de bebés prematuros y que comprende la estimulación de diferentes áreas relacionadas con la manera en que los padres interactúan con sus hijos, para promover sus habilidades comunicativas. También, analizar el efecto de dicha intervención en el desarrollo del lenguaje evaluado, mediante una prueba estandarizada, al año de edad. Hasta el presente no se dispone de información acerca de una investigación de este tipo en México. 


\section{Método}

Este estudio contó con un enfoque cuantitativo, con un diseño longitudinal. El alcance pretende ser explicativo pero, dado que no se contó con un grupo control, se considera un pre-experimento con un solo grupo, el cual cuenta con evaluaciones pre intervención y post intervención (Sackett \& Mullen, 1993).

\section{Participantes}

En el estudio participaron 21 bebés prematuros (12 niñas y 9 niños), con una edad gestacional promedio de 30 semanas (+/-2.28), que acudieron a la Unidad de Investigación en Neurodesarrollo "Dr. Augusto Fernández Guardiola", del Instituto de Neurobiología de la Universidad Nacional Autónoma de México, Campus Juriquilla, Querétaro. Los bebés presentaron distintos factores de riesgo de daño cerebral y entre los más frecuentes estaban la hiperbilirrubinemia $(85.7 \%$ ) y la sepsis neonatal $(80.9 \%)$. Los bebés ingresaron al protocolo de investigación de la Unidad mencionada, dirigido básicamente a la prevención de la parálisis cerebral, mediante la Terapia Neurohabilitatoria (Harmony et al., 2016), aproximadamente a los 3 meses de edad corregida. Todos los bebés participantes habían presentado signos de Leucomalacia Periventricular Difusa (LPD) en sus imágenes por resonancia magnética.

La técnica de muestreo fue no probabilística por cuotas. Se hizo una invitación a las familias asistentes a la Unidad y se incluyeron en el estudio a aquellas que cumpliesen con los criterios de inclusión. Se les brindó atención, también, a las familias que no cumpliesen con dichos criterios, aunque sus datos no formaron parte del presente trabajo. No se incluyeron en esta investigación a familias cuyos bebés presentasen daño cerebral distinto al de LPD o que presentasen deficiencias sensoriales auditivas o visuales. Tampoco se aceptaron datos de familias en las que los padres tuviesen una escolaridad menor a primaria terminada. Se obtuvo el consentimiento informado de los padres de los bebés y se trató la información personal de los participantes de manera confidencial.

\section{Procedimiento}

Programa de entrenamiento a los padres. Se diseñó un programa de intervención temprana basado en un entrenamiento para los padres acerca del desarrollo del lenguaje y la manera en la que se puede estimular. Se escogió esta forma de entrenamiento ya que, como se señaló anteriormente, los programas de intervención temprana que se enfocan en el esfuerzo conjunto de padres, madres y bebés, son más efectivos que los dirigidos solamente al bebé (Barrera, et al., 1986; Shonkoff \& Hauser-Cram, 1987). Por tanto, se eligió un entrenamiento dirigido a los padres como principal objetivo de las sesiones, porque se esperaba que lo que aprendieran durante ellas pudieran llevarlo a cabo también en sus casas.

En este programa de intervención los lactantes asistieron, junto con sus padres, a un total de 12 sesiones de una hora de duración. En los primeros 30 minutos de la sesión se instruyó a los padres acerca de diversos temas relacionados con las habilidades comunicativas de los infantes. Los siguientes 30 minutos, la terapeuta hacía demostraciones de cómo podían estimular esas habilidades comunicativas en sus hijos, estimulando conductas vocales y verbales en los lactantes. 
Los padres recibieron información acerca de las siguientes áreas: adquisición y desarrollo del lenguaje en niños sanos, reconocimiento y sensibilización a las respuestas de los niños, modificación del ambiente en el que el niño se desenvuelve en casa para favorecer una estimulación óptima, habla dirigida al niño (también llamada materlalia y que ha mostrado mejorar la percepción del lenguaje en niños, según autores como Liu, et al., 2009; Tsao et al., 2004) y otras estrategias verbales, estimulación auditiva y prácticas de alfabetización inicial (lectura de cuentos).

También, se estructuró un método para medir la eficacia de este programa de entrenamiento por medio de un cuestionario en el que se les hacen preguntas a los padres acerca de los temas que serían tratados durante el entrenamiento. El cuestionario fue diseñado por los autores de este trabajo. Dicho cuestionario estaba conformado por 35 preguntas, divididas en las siguientes categorías: desarrollo general (2), desarrollo de la comprensión (2), reconocimientos de las necesidades del bebé (4), ambiente adecuado para la estimulación (5), el lenguaje infantil (4), atención auditiva (5), estrategias verbales (10) y aspectos generales (3). Las respuestas se daban en una escala tipo Likert para ver qué tanto habían aprendido los padres durante las sesiones.

Los valores oscilaban entre 1 y 5 . Se determinó la validez de contenido del instrumento a través de un juicio de expertos y la confiabilidad se evaluó a través del cálculo del alfa de Cronbach. Se obtuvo un valor de alfa de .65 para el instrumento completo. Los padres llenaron el cuestionario antes de iniciar las sesiones de terapia. Al término de las 12 sesiones, se volvió a aplicar el cuestionario para comparar las respuestas.

\section{Evaluación del lenguaje}

Para evaluar el lenguaje de los bebés a los 12 meses, se utilizó el Inventario del Desarrollo de las Habilidades Comunicativas McArthur (IDHC, Jackson-Maldonado, et al., 2003). El inventario mide la comprensión, producción y el uso de gestos; ha sido usado ampliamente en diversas investigaciones.

La versión del inventario utilizada fue la adaptada y estandarizada en población mexicana y tiene datos de confiabilidad test-retest de entre .63 y .97 , así como una validez predictiva de .70 y una validez de criterio de .84 (Jackson-Maldonado, Thal, Marchman, Bates, \& Gutierrez-Clellen, 1993). El instrumento ha mostrado ser un buen estimador del lenguaje de los niños a pesar de ser un reporte paterno (Mayor \& Plunkett, 2011).

A los tres años de edad se aplicó, a 14 niños de la muestra, la Preschool Language Scale Fifth Edition (PLS-5) (Zimmerman, Steiner, \& Pond, 2012a), la cual evalúa el lenguaje receptivo y el expresivo. Esta prueba se ha usado como auxiliar en el diagnóstico de alteraciones de lenguaje, tiene una sensibilidad de .85 y una especificidad de .88 , una validez concurrente de .76 y una confiabilidad de consistencia interna de entre .80 y .94 (Zimmerman, Steiner, \& Pond, 2012b).

Por lo anterior, se considera que es una prueba adecuada para evaluar lenguaje en estas edades. Los resultados de las evaluaciones de lenguaje solo se llevaron a cabo después de la intervención, debido a que los bebés iniciaron el programa de intervención a una edad muy temprana en la que no era posible todavía obtener datos de lenguaje. Por tanto, los 
datos obtenidos con esta prueba solo se utilizarán con un propósito ilustrativo y complementario a los encontrados con el IDHC.

Estrategia de análisis

Se promediaron los valores de las respuestas del cuestionario para cada una de las áreas evaluadas y del total de respuestas de cada padre, antes de comenzar la intervención temprana y después de concluirla. Posteriormente, se aplicó la prueba de rangos de Wilcoxon para determinar si existían diferencias significativas entre las respuestas de los padres en los dos momentos en que se aplicó el cuestionario. Para evaluar los resultados obtenidos, tanto en el IDHC como en la PLS-5, se tuvieron en cuenta los puntajes estandarizados que ofrecen ambos instrumentos.

\section{Resultados}

Los resultados obtenidos muestran la comparación de las respuestas obtenidas en el cuestionario aplicado a los padres antes y después de las sesiones de entrenamiento y se realizó la comparación estadística entre los dos momentos. Se encontraron diferencias significativas, en casi todas las áreas evaluadas con el cuestionario, como se muestra en la tabla 1. Los puntajes más altos corresponden al final del entrenamiento, excepto en Ambiente y la de Aspectos Generales, la cual no se incluyó en las figuras. En la figura 1 y 2 se muestran los promedios de las respuestas dadas por los padres en los cuestionarios aplicados.

Al mismo grupo de bebés prematuros, al año de edad corregida, se le aplicó el Inventario del Desarrollo de las Habilidades Comunicativas MacArthur (Jackson-Maldonado et al., 2003) para evaluar el desarrollo del lenguaje, particularmente en lo referente a la "Comprensión de palabras" y "Producción de palabras", que constituyen subpruebas de dicho Inventario. La mayoría de los bebés $(20 / 21=95.2 \%)$ obtuvo percentiles por encima del valor 30 en la subprueba de "Comprensión de palabras".

El percentil 10 se considera el punto de corte de la prueba para clasificar un bebé como normal en esta variable. Todos los participantes obtuvieron puntajes por encima de este percentil; las puntuaciones oscilaron entre el percentil 25 y 99. En la subprueba

Tabla 1

Comparación, mediante la prueba de rangos de Wilcoxon, de los valores obtenidos en las diferentes áreas del cuestionario antes después del entrenamiento

\begin{tabular}{lcccc}
\hline \multicolumn{1}{c}{ Área } & Antes $(\bar{X})$ & Después $(\bar{X})$ & Valor Z & $p$ \\
\hline Desarrollo de la comprensión & 6.04 & 9.09 & -3.935 & .000 \\
Estímulos Verbales & 38 & 40.71 & -1.956 & .050 \\
Lenguaje Infantil & 13.19 & 15.47 & -2.556 & .011 \\
Atención Auditiva & 21.14 & 23.61 & -2.953 & .003 \\
Ambiente & 16.61 & 18.42 & -1.805 & .071 \\
Necesidades & 15.66 & 17.38 & -3.456 & .001 \\
Desarrollo General & 7.52 & 8.57 & -2.539 & .011 \\
Total & 130.61 & 145.33 & -3.616 & .000 \\
\hline
\end{tabular}



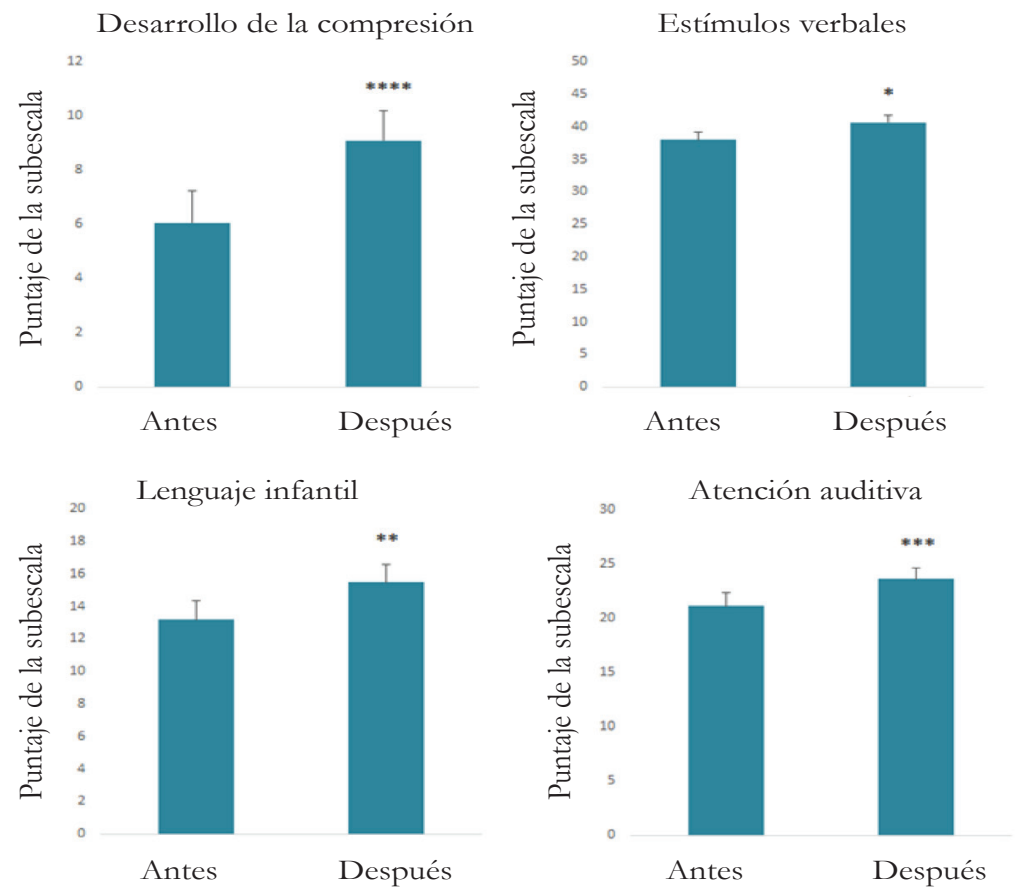

Figura 1. Puntajes promedio obtenidos en las áreas de Desarrollo de la Comprensión, Estímulos Verbales, Lenguaje Infantil y Atención Auditiva. En todas se encontraron diferencias significativas antes y después del entrenamiento a padres $\left(* \mathrm{p} \leq .05 ;{ }^{* *} \mathrm{p} \leq .01 ; * * * \mathrm{p} \leq .003 ;{ }^{* * * *} \mathrm{p} \leq .000\right)$.
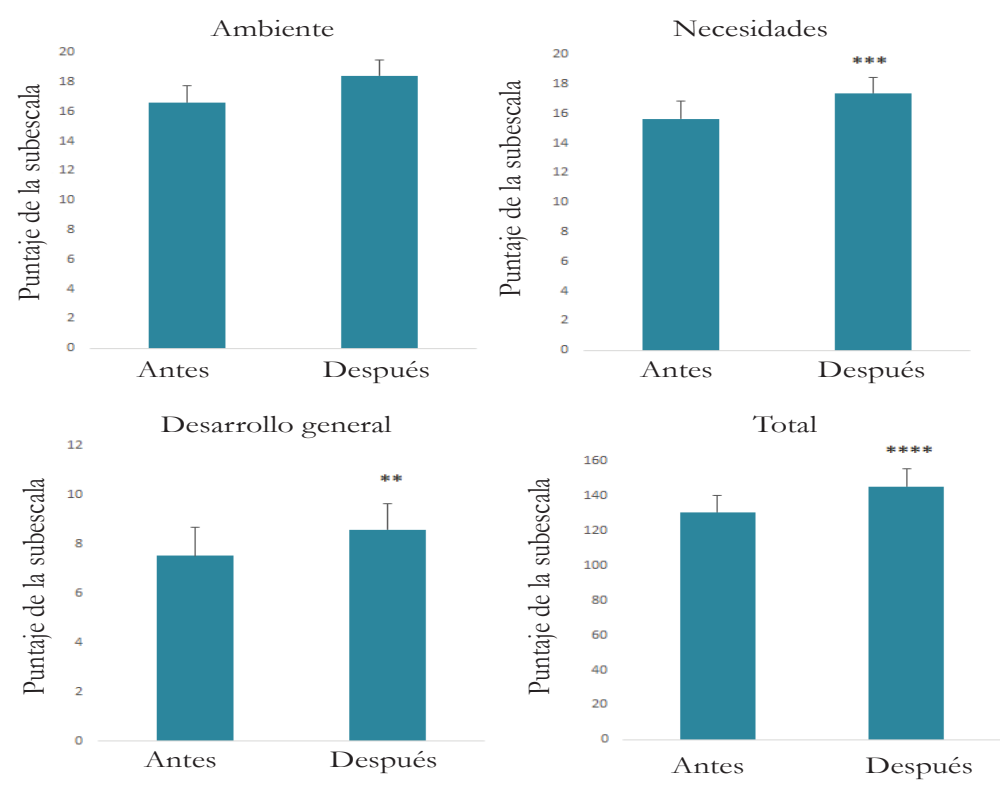

Figura 2. Puntajes promedio obtenidos en las áreas de Ambiente, Necesidades, Desarrollo General y el Total. Se muestran las diferencias significativas observadas en tres de las áreas evaluadas, antes y después del entrenamiento a padres (** $\mathrm{p} \leq .01 ; * * * \mathrm{p} \leq .003 ; * * * * \mathrm{p} \leq .000)$. 
"Producción de palabras", todos los bebés obtuvieron un percentil igual o mayor a 10 y sus percentiles oscilaron entre 10 y 90 .

También, se llevó a cabo un seguimiento de estos bebés y a los 3 años se les aplicó la prueba de lenguaje SPLS-5 a 14 niños que ya habían cumplido esa edad (Zimmerman et al, 2012a). La mayoría $(13 / 14=92.85 \%$ ) obtuvo un puntaje considerado como normal en lo receptivo, expresivo y el total de lenguaje.

Los puntajes estandarizados para la subprueba de Comprensión Auditiva oscilaron, en la muestra, entre 85-129, para la subprueba de Expresión entre 67-125 y para el Total entre 74-123. La media para esta prueba es de 100 y la desviación estándar es de 15. Uno de los bebés que obtuvo los bajos puntajes en el IDHC, tanto en comprensión como en producción del lenguaje, es el que también mostró un bajo puntaje en el PLS-5; fue prematuro extremo (29 semanas de gestación) y tuvo problemas de reflujo, por lo que era muy complicado alimentarlo adecuadamente y tenía bajo peso.

\section{Discusión}

En un estudio previo, Harmony et al. (2016) confirmaron que la Terapia Neurohabilitatoria disminuye las secuelas neurológicas y cognitivas en niños prematuros y de término con daño cerebral perinatal demostrado por evaluaciones clínicas, de neurodesarrollo e imágenes por resonancia magnética. En el presente trabajo se comprobaron, en un grupo de prematuros con riesgo de daño cerebral perinatal sometidos a Terapia Neurohabilitatoria, los beneficios específicos sobre la adquisición del lenguaje derivados del entrenamiento ofrecido a los padres para aplicarlo con sus bebés en el primer año de vida y de forma sostenida durante este tiempo.

Como se señaló en la introducción de este artículo, los estudios comparativos acerca de la adquisición temprana del lenguaje en bebés prematuros y de término, realizados generalmente en pre-escolares y escolares, han reportado hallazgos controversiales; por un lado, unos reportan un retraso en sus habilidades de lenguaje, comparados con bebés nacidos a término (Sansavini et al., 2006; Foster-Cohen et al., 2007; Kern \& Gayrau, 2007; Ortiz-Mantilla et al., 2008; Wolke et al., 2008; Guarini et al., 2009; Stolt et al., 2009; Stolt et al., 2011); por otro lado, otros no han encontrado diferencias significativas al comparar ambos grupos (Cattani et al., 2010; Pérez-Pereira \& Resches, 2011) cuando se hace una corrección de la edad y se iguala el nivel cognoscitivo.

Es difícil decidir cuál de estos dos tipos de hallazgos es el correcto, pero lo que sí sugieren los resultados del presente trabajo es que es posible encontrar puntuaciones normales cuando se utilizan pruebas estandarizadas y se lleva a cabo la corrección por la edad, teniendo una variable interventora en el proceso.

Tomando en cuenta lo anterior, un aporte que se considera muy importante es haber iniciado la terapia muy tempranamente, ya que la mayoría de los programas destinados a estimular el lenguaje están dirigidos a niños de mayor edad; esto es, cuando ya se ha comprobado un retraso en la adquisición de las habilidades comunicativas o por lo menos cuando no se ha desarrollado el lenguaje expresivo y esto empieza a preocupar a los padres (Moreno-Flagge, 2013). 
Otro aspecto relevante es haber incluido a los padres como los principales promotores para el desarrollo del lenguaje, ya que el entrenamiento brindado estos puede funcionar como medida preventiva para que sus bebés prematuros no tengan dificultades posteriores en la adquisición del lenguaje.

En opinión de los autores de este trabajo, si se logra que los padres se comuniquen regularmente con sus bebés, a través del juego y las actividades diarias, se puede ayudar a estos últimos a entender el significado de las palabras y las frases mucho antes de que pronuncien su primera palabra.

En el estudio actual, al año de edad, los resultados del IDHC evidenciaron que la mayoría de los bebés mostraron puntajes dentro de lo esperado para su edad tanto en comprensión como en producción del lenguaje. Además, aunque los resultados de la PLS-5 administrada a los 3 años deben ser tomados con cautela dado que la prueba no tuvo lógicamente una aplicación inicial y solo incluyó a 14 de los 21 bebés evaluados, sus hallazgos sugieren puntajes de acuerdo con la edad en el lenguaje receptivo y expresivo.

Se ha reportado en otros estudios que las estrategias de interacción que tienen los padres con sus hijos afectan el desarrollo de su lenguaje y que, por lo tanto, el entrenamiento a los padres de niños con dificultades en el lenguaje resulta ser una herramienta efectiva de intervención temprana (Kaiser \& Roberts, 2012). En la presente investigación quedó evidenciado, también, que gracias la participación activa de los padres mediante el programa de entrenamiento utilizado, pueden obtenerse resultados satisfactorios en el desarrollo del lenguaje de sus hijos, por lo menos hasta los 3 años, que es una edad en la que se espera que su lenguaje sea bastante inteligible, que ya hayan adquirido correctamente la estructura gramatical de su lengua materna y que sean capaces de comunicarse no solamente con sus familiares sino también con personas fuera de su núcleo familiar.

Es importante tener presente que los bebés prematuros de esta muestra, si bien tuvieron los beneficios de la Terapia Neurohabilitatoria en el primer de año de vida, también se enfrentaron a los efectos adversos producidos por los factores de riesgos de daño perinatal que presentaban, así como las consecuencias de su prematurez mostradas por la imagenología (signos de Leucomalacia Periventricular Difusa).

La repercusión de estos antecedentes en el desarrollo del lenguaje fue evidenciada en el trabajo de Avecilla et al. (2012), quienes reportaron en una muestra similar a la de este trabajo -pero en la que los padres no tuvieron un entrenamiento sistemático para promover la adquisición del lenguaje de sus bebés prematuros-, que los sujetos evaluados presentaban bajos puntajes en el IDHC al año de edad. Por tanto, los resultados obtenidos en este trabajo parecen ofrecer una nueva oportunidad a estos bebés para que adquieran un lenguaje dentro de límites normales si se aplica el entrenamiento propuesto.

Para futuras investigaciones dentro de este ámbito, se sugiere validar los resultados de este trabajo en otra muestra independiente; complementar la evaluación del entrenamiento a los padres con otras medidas, tales como el registro de la actividad cerebral relacionado con sonidos del lenguaje, observaciones directas de la interacción padre-hijo (documentación con video), así como un seguimiento a largo plazo para ver qué sucede con el lenguaje de los niños que se estudien con el entrenamiento a padres utilizado en esta investigación 


\section{Agradecimientos}

Las autoras agradecen la valiosa colaboración de las especialistas en Comunicación Humana, Claudia Jiménez, Amaya Soberón y Johanna Flores, por su apoyo con la aplicación e interpretación de la PLS-5; así como de los profesionales de la Unidad de Investigación en Neurodesarrollo y del Instituto de Neurobiología, sobre todo los de las Áreas de Lenguaje y de la Terapia Neurohabilitatoria sin cuya colaboración no hubiera sido posible realizar este trabajo: Vanessa Monge, Ma. E. Mónica Carlier, Ma. Elena Juárez, Jesús Barrera, Consuelo Pedraza, Cristina Carrillo, Héctor Belmont, Ma. de Lourdes Ayala, Leonor Casanova, Ramón Martínez. Agradecen, además, el apoyo que la autora principal recibió como becaria del CONACYT y de los proyectos CONACYT 166772, 218556 y PAPIIT IN200917.

\section{Referencias}

Avecilla-Ramírez, G., Harmony, T., Porras-Kattz, E., Ricardo-Garcell, J., FernándezBouzas, A., \& Santiago, E. (2012). Indicadores electrofisiológicos de la percepción fonética en lactantes con riesgo de trastornos del lenguaje.Ciencia@ $U A Q, 3(1), 14-26$.

Barrera, M., Rosenbaum, P., \& Cunningham, C. (1986). Early home intervention with low-birth-weight infants and their parents. Child Development, 57(1), 20-33. doi: $10.2307 / 1130634$

Cattani, A., Bonifacio, S., Fertz, M., Iverson, J., Zocconi, E., \& Caselli, C. (2010). Communicative and linguistic development in preterm children: a longitudinal study from 12 to 24 months. International Journal of Language \& Communication Disorders, 45(2), 162-173.

Conboy, B., Brooks, R., Meltzoff, A., \& Kuhl, P. (2015). Social Interaction in Infants' Learning of Second-Language Phonetics: An Exploration of Brain-Behavior Relations. Developmental Neuropsychology, 40(4), 216-229. doi:_10.1080/87565641 .2015 .1014487

Doyle. L., \& Victorian Infant Collaborative Study Group. (2001). Outcome at 5 years of age of children 23 to 27 weeks' gestation: refining the prognosis. Pediatrics, 108(1), 134-141.

Fernald, A. (1993). (coord.). Vocalizations to infants as biologically relevant signals, en P. Bloom Language Acquisition Core Readings. Wheatsheaf: Harvester.

Foster-Cohen, S., Edgin, J, Champion, P., \& Woodward, L. (2007). Early delayed language development in very preterm infants: evidence from the MacArthur-Bates CDI. Journal of Child Language, 34(3), 655-675. doi: 10.1017/S0305000907008070

Goldstein, M., King, A., \& West, M. (2003). Social interaction shapes babbling: Testing parallels between birdsong and speech. Proceedings of the National Academy of Sciences, 100(13), 8030-8035. doi: 10.1073/pnas.1332441100 
Guarini, A., Sansavini, A., Fabbri, C., Alessandroni, R., Faldella, G., \& KarmillofSmith, A. (2009). Reconsidering the impact of preterm birth on language outcome. Early Human Development, 85(10), 639-645. doi: 10.1016/j. earlhumdev.2009.08.061

Guralnick, M. J. (2008). International perspectives on early intervention: A search for common ground. Journal of Early Intervention, 30(2), 90-101.

Guralnick, M. (2012). Preventive interventions for preterm children: Effectiveness and developmental mechanisms. Journal of Developmental \& Behavioral Pediatrics, 33(4), 352-364. doi: 10.1097/DBP.0b013e31824eaa3c

Harmony, T., Barrera-Reséndiz, J., Juárez-Colín, M., Carrillo-Prado, C., Pedraza-Aguilar, M., Asprón, A., Hinojosa, M., Fernández, T., \& Ricardo-Garcell., J. (2016). Longitudinal study of children with perinatal brain damage in whom early neurohabilitation was applied: Preliminary report. Neuroscience Letters, 611, 59-67. doi: 10.1016/j.neulet.2015.11.013

Horwood, J., Mogridge, N., \& Darlow, B. (1998). Cognitive, educational, and behavioural outcomes at 7 to 8 years in a national very low birthweight cohort. Archives of Disease in Childhood-Fetal and Neonatal Edition, 79(1), 12-20.

Jackson-Maldonado, D., Thal, D., Marchman, V., Bates, E., \& Gutierrez-Clellen, V. (1993). Early lexical development in Spanish-speaking infants and toddlers. Journal of Child Language, 20(3), 523-549. doi; 10.1017/S0305000900008461

Jackson-Maldonado, D., Thal, D., Marchman, V., Newton, T., Fenson, L., \& Conboy, B. (2003). User's Guide and Technical Manual Contents. MacArthur Inventarios. PaulBrookes Publishing Co.

Jiménez, A., Servera, C., Roca, A., Frontera, G., \& Pérez, J. (2008). Seguimiento de recién nacidos de peso menor o igual a $1.000 \mathrm{~g}$ durante los tres primeros años de vida. Anales de Pediatría, 68(4), 320-328. doi: 10.1157/13117701

Kaiser, A., \& Roberts, M. (2012). Advances in Early Communication and Language Intervention. Journal of Early Intervention. 33(4), 298-309. doi : $10.1177 / 1053815111429968$

Kern, S., \& Gayraud, F. (2007). Influence of preterm birth on early lexical and grammatical acquisition. First Language, 27(2), 159-73. doi: 10.1177/0142723706075790

Kuhl, P. (2007). Is speech learning "gated” by the social brain? Developmental Science, 10(1), 110-120. doi: 10.1111/j.1467-7687.2007.00572.x

Kuhl, P. (2014). Early Language Learning and the Social Brain. Cold Spring Harbor Symposia on Quantitative Biology, 79, 211-220. doi: 10.1101/sqb.2014.79.024802

Liu, H., Tsao, F., \& Kuhl, P. (2009). Age-related changes in acoustic modifications of Mandarin maternal speech to preverbal infants and five-year-old children: a longitudinal study. Journal of Child Language, 36(4), 909-922. doi: 10.1017/ S030500090800929X 
Magill-Evans, J., \& Harrison, M. (1999). Parent-child interactions and development of toddlers born preterm. Western Journal of Nursing Research, 21(3), 292-307. doi: $10.1177 / 01939459922043893$

Magill-Evans, J., \& Harrison, M. (2001). Parent-child interactions, parenting stress, and developmental outcomes at 4 years. Children's Health Care, 30(2), 135-150. doi: 10.1207/S15326888CHC3002_4

Mayor, J., \& Plunkett, K. (2011). A statistical estimate of infant and toddler vocabulary size from CDI analysis. Developmental Science, 14(4), 769-785. doi: 10.1111/j.14677687.2010.01024.x

Menyuk, P., Chesnick, M., Liebergot, J., Korngold, B., D’Agostino, R., \& Belanger, A. (1991). Predicting Reading Problems in At-Risk Children. Journal of Speech, Language, and Hearing Research, 34(4), 893-903. doi: 10.1044/jshr.3404.893

Moreno-Flagge, N. (2013). Trastornos del lenguaje. Diagnóstico y tratamiento. Revista de Neurología, 57(1), 85-94.

Ortiz-Mantilla, S., Choudhury, N., Leevers, H., \& Benasich, A. (2008). Understanding language and cognitive deficits in very low birth weight children. Developmental Psychobiology, 50(2), 107-126. doi: 10.1002/dev.20278

Pérez-Pereira, M., \& Resches, M. (2011). Concurrent and predictive validity of the Galician CDI. Journal of Child Language, 38(1), 121-140. doi: 10.1017/S0305000909990262

Resnick, M., Eyler, F., Nelson, R., Eitzman, D., \& Bucciarelli, R. (1987). Developmental intervention for low birth weight infants: Improved early developmental outcome. Pediatrics, 80(1), 259-283.

Roberts, M., \& Kaiser, A. (2011). The Effectiveness of Parent-Implemented Language Interventions: A Meta-Analysis. American Journal of Speech-Language Pathology, 20(3), 180-199. doi: 10.1044/1058-0360(2011/10-0055)

Sackett, P., \& Mullen, E. (1993). Beyond formal experimental design: Towards an expanded view of the training evaluation process. Personnel Psychology, 46(3), 613627. doi: 10.1111/j.1744-6570.1993.tb00887.x

Sansavini, A., Guarini, A., Alessandroni, R., Faldella, G., Giovanelli. G., \& Salvioli, G. (2006). Early relations between lexical and grammatical development in very immature Italian preterms. Journal of Child Language, 33(1), 199-216. doi: 10.1017/ S0305000905007208

Shonkoff, J., \& Hauser-Cram, P. (1987). Early intervention for disabled infants and their families: A quantitative analysis. Pediatrics, 80(5), 650-658.

Snow, C. (1997). Mother's speech research: from input to interaction. In: Talking to Children. Cambridge: Cambridge University Press.

Stolt, S., Haataja, L., Lapinleimu, H., \& Lehtonen, L. (2009). The early lexical development and its predictive value to language skills at 2 years in very-lowbirth-weight 
children. Journal of Communication Disorders, 42(2), 107-123. doi: 10.1016/j. jcomdis.2008.10.002

Stolt S., Lehtonen, L., Haataja, L., \& Lapenleimu, H. (2011). El lenguaje utilizado por niños con muy bajo peso al nacer en la interacción temprana entre madre e hijo, con atención especial a la aparición de la gramática. Revista de Logopedia, Foniatría y Audiología, 31(3), 115-124. doi: 10.1016/S0214-4603(11)70180-2

Tallal, P., \& Gaab, N. (2006). Dynamic auditory processing, musical experience and language development. Trends in Neurosciences, 29(7), 382-390. doi: 10.1016/j. tins.2006.06.003

Tsao, F., Liu, H., \& Kuhl, P. (2004). Speech Perception in Infancy Predicts Language Development in the Second Year of Life: A Longitudinal Study. Child Development, 75(4), 1067-1084. doi: 10.1111/j.1467-8624.2004.00726.x

Ward, S. (1999). An investigation into the effectiveness of an early intervention method for delayed language development in young children. International Journal of Language and Communication Disorders, 34(3), 243-264. doi: 10.1080/136828299247405

Wolke, D., Samara, M., Bracewell, M., Marlow, N. (2008). Specific Language Difficulties and School Achievement in Children Born at 25 Weeks of Gestation or Less. The Journal of Pediatrics, 152(2), 256-262. doi: 10.1016/j.jpeds.2007.06.043

Zauche, L., Thul, T., Darcy, A., \& Staple-Wax, J. (2016). Influence of language nutrition on children's language and cognitive development: An integrated review. Early Childhood Research Quarterly, 36, 318-333. doi: 10.1016/j.ecresq.2016.01.015

Zimmerman, I., Steiner, V., \& Pond, R. (2012a). Preschool Language Scale- Fifth Edition. United States of America, Pearson.

Zimmerman, I., Steiner, V., \& Pond, R. (2012b). Test review: Preschool Language ScalesFifth Edition (PLS-5) Spanish. Leaders, 1-18. Retrieved from https:/ / cpb-us-w2. wpmucdn.com/edblogs.columbia.edu/dist/d/730/files/2015/05/PLS-5Spanish-2012_Pub_10.29.13-18cdkt8.pdf

Recibido: 12 de octubre de 2017

Aceptado: 17 de abril de 2018 